Y. C. Minh and E. F. van Dishoeck, eds.

\title{
The Fractional Ionization in Molecular Cloud Cores
}

\author{
Paola Caselli \\ Osservatorio Astrofisico di Arcetri, Largo E. Fermi 5, I-50125 Firenze, \\ Italy
}

Abstract. Ions and electrons play a key role in the chemical and dynamical evolution of interstellar clouds. Gas phase ion-molecule reactions are major chemical routes to the formation of interstellar molecules. The ionization degree determines the coupling between the magnetic field and the molecular gas through ion-neutral collisions, and thus regulates the rate of star formation. In the theoretical determination of the degree of ionization we run into several sources of uncertainty, including the poorly known cosmic ray flux and metal depletion within the cores, the penetration of UV radiation deep into regions of high visual extinction due to cloud inhomogeneities, and the ionization rate increase in the proximity of young stellar objects which may be strong X-ray emitters. Observational estimates of electron (or ion) fractions $x(e)\left(\equiv n(e) / n\left(\mathrm{H}_{2}\right)\right.$, where $n(e)$ and $n\left(\mathrm{H}_{2}\right)$ are the electron and molecular hydrogen number densities, respectively) in dense cloud cores are thus of considerable interest. In this paper, I will review recent improvements in the estimates of the ion fraction in dense cores and point out the difficulties in determining $x(e)$.

\section{Introduction}

A direct measure of electron abundances is hindered by the difficulty of observing $\mathrm{H}_{3}^{+}$, one of the most abundant molecular ions, which has recently been detected in dense clouds (McCall et al. 1999), and atomic species with low ionization potentials, such as atomic carbon and metals, which may be the main repositories of positive charge. Rather, indirect determinations of $n(e)$ through the estimate of molecular column densities sensitive to electron densities and the application of chemical models are used. There have been several attempts in the past to use either the observed degree of deuterium fractionation (e.g. Guélin et al. 1982; Wootten et al. 1982) or the abundance of selected molecular ions (de Boisanger et al. 1996) to determine the electron fraction.

Recent improvements in the laboratory measurements of the $\mathrm{H}_{3}^{+}$and $\mathrm{H}_{2} \mathrm{D}^{+}$ dissociative recombination rates (Larsson et al. 1996), crucial in chemical models, as well as the increased sensitivity, spectral, and spatial resolution of millimeter telescopes, allowed us to refine previous determinations of $x(e)$ (Caselli et al. 1998, hereafter CWTH; Williams et al. 1998; Bergin et al. 1999; Anderson et al. 1999) and has furnished a new impetus for more detailed investigations of the chemical and physical properties of cloud cores. I will review recent im- 
provements in the estimates of the ion fraction in dense cores (Sect. 2 and 3). I will also present a new observational study of L1544, a starless core in Taurus, where asymmetric line profiles and the large gas column density suggest that the core is in a very early phase of star formation (Sect. 4). The main sources of uncertainty in $x(e)$ and possible ways to eliminate them are listed in Sect. 5.

\section{Analytic Estimates of $x(e)$}

Observations of species which are abundant, relatively easy to detect, and directly related to the electron abundance, are needed to measure the fractional ionization in dense clouds. The two molecular ions $\mathrm{HCO}^{+}$and $\mathrm{DCO}^{+}$fulfil the above requirements and a simple chemical scheme allows one to put limits on $x(e)$ from measurements of their abundance ratio (e.g. Watson 1977; Wootten et al. 1979; Guélin et al. 1982).

The main production and destruction mechanisms for $\mathrm{HCO}^{+}$and $\mathrm{DCO}^{+}$ are shown in Figure 1. In dark clouds, the cosmic ray ionization of molecular hydrogen, followed by the reaction $\mathrm{H}_{2}^{+}+\mathrm{H}_{2} \rightarrow \mathrm{H}_{3}^{+}+\mathrm{H}$, leads to the formation of $\mathrm{H}_{3}^{+}$, which plays a crucial role in gas-phase chemistry (e.g. Herbst \& Leung 1989). The molecular ion $\mathrm{HCO}^{+}$is formed through the reaction between $\mathrm{H}_{3}^{+}$and $\mathrm{CO}$ (the most abundant molecule in dense clouds after $\mathrm{H}_{2}$ ), and is destroyed by electrons. The formation of deuterated molecules begins with the isotope exchange reaction $\mathrm{H}_{3}^{+}+\mathrm{HD} \rightleftharpoons \mathrm{H}_{2} \mathrm{D}^{+}+\mathrm{H}_{2}+\Delta E$, which is exothermic by $\Delta E \simeq 230 \mathrm{~K}$ (e.g. Millar et al. 1989) so that at the low temperatures typical of dark clouds the reverse reaction is inhibited and deuterium fractionation can proceed. $\mathrm{DCO}^{+}$is then produced by the reaction between $\mathrm{CO}$ and $\mathrm{H}_{2} \mathrm{D}^{+}$and destroyed by dissociative recombination. The $\left[\mathrm{DCO}^{+}\right] /\left[\mathrm{HCO}^{+}\right]$abundance ratio in cold dense cores is typically about 3 orders of magnitude larger than the interstellar $[\mathrm{D}] /[\mathrm{H}]$ ratio $\left(\sim 1.5 \times 10^{-5}\right.$; Linsky et al. 1995) and this reflects the enhancement of $\mathrm{H}_{2} \mathrm{D}^{+}$relative to $\mathrm{H}_{3}^{+}$.

There have been several recent discussions of the value of the rate coefficients for the dissociative recombination of $\mathrm{H}_{3}^{+}$and $\mathrm{H}_{2} \mathrm{D}^{+}\left(\alpha_{1}\right.$ and $\alpha_{2}$ in Fig. 1) which are crucial for the chemistry and the ionization balance of molecular clouds. These rate coefficients have been recently re-measured (Larsson et al. 1996) and the new values have been used to refine previous estimates of $x(e)$ (e.g. CWTH). For the simple chemical system in Fig. 1, assuming steady state, and substituting the numerical values of the rate coefficients, the following relations determining $x(e)$ and the cosmic ray ionization rate $\zeta$ are obtained $(\mathrm{CWTH})$ :

$$
\begin{aligned}
x(e) & =\frac{2.7 \times 10^{-8}}{\left[\mathrm{DCO}^{+}\right] /\left[\mathrm{HCO}^{+}\right]}-\frac{1.2 \times 10^{-6}}{f_{\mathrm{D}}} \\
\zeta & =\left[7.5 \times 10^{-4} x(e)+\frac{4.6 \times 10^{-10}}{f_{\mathrm{D}}}\right] x(e) n\left(\mathrm{H}_{2}\right) \frac{\left[\mathrm{HCO}^{+}\right]}{[\mathrm{CO}]},
\end{aligned}
$$

where $f_{\mathrm{D}}$ is the depletion factor, and $1 / f_{\mathrm{D}}$ is defined as the fraction of $\mathrm{CO}$ and $\mathrm{O}$ - the most important destruction partners of $\mathrm{H}_{3}^{+}$and $\mathrm{H}_{2} \mathrm{D}^{+}$- frozen onto dust grains. This simple model demonstrates the importance of an independent determination of $f_{\mathrm{D}}$ if one wishes to estimate $x(e)$. Note that equation 1 cannot account for $\left[\mathrm{DCO}^{+}\right] /\left[\mathrm{HCO}^{+}\right]$abundance ratios larger than $0.023 \times f_{\mathrm{D}}$. Based 


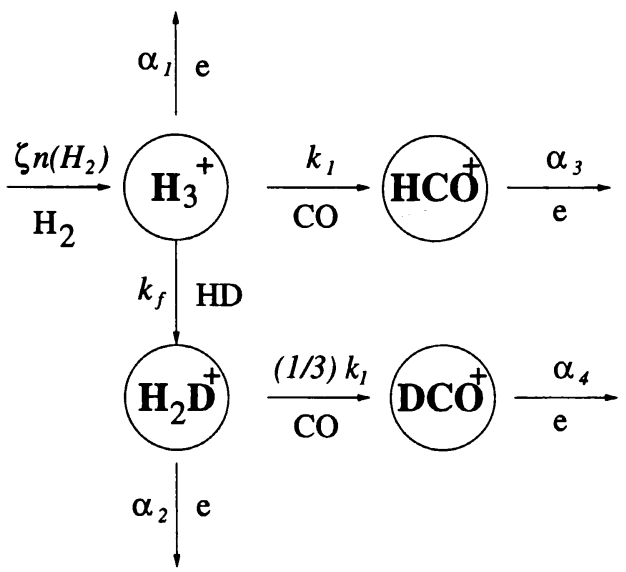

Figure 1. Schematic representation of the main production and destruction mechanisms for $\mathrm{HCO}^{+}$and $\mathrm{DCO}^{+}$. The $\alpha$ 's and $k$ 's are the corresponding rate coefficients (cf. Caselli et al. 1998). The $\left[\mathrm{DCO}^{+}\right] /\left[\mathrm{HCO}^{+}\right]$abundance ratio is directly related to the electron abundance.

on the Butner et al. (1995) data (see next Section), one needs $f_{\mathrm{D}}>1$ for the majority of sources.

\section{Determination of $x(e)$ via Detailed Chemical Models}

Detailed chemical models can be used to reproduce the observed $\left[\mathrm{DCO}^{+}\right] /\left[\mathrm{HCO}^{+}\right]$ and $\left[\mathrm{HCO}^{+}\right] /[\mathrm{CO}]$ abundance ratios and from them deduce the electron fraction, the cosmic ray ionization rate, and abundance of other species. CWTH have computed models of the evolution of the chemistry in a dense core (kinetic temperature $\left.T=10 \mathrm{~K}, n\left(\mathrm{H}_{2}\right) \sim 10^{4}-10^{5} \mathrm{~cm}^{-3}\right)$ using the "New Standard Model" of Lee et al. (1996) modified in order to consider simple deuterated species. In particular, reactions with atomic deuterium (e.g. $\left.\mathrm{HCO}^{+}+\mathrm{D} \rightarrow \mathrm{DCO}^{+}+\mathrm{H}\right)$ have been included, following Dalgarno \& Lepp (1984).

Free parameters in these models are: (i) $f_{\mathrm{D}}$, the depletion factor; (ii) $\sum x\left(M_{i}^{+}\right)$, the abundance of refractory metals, such as $\mathrm{Fe}, \mathrm{Mg}$, and $\mathrm{Na}$, initially present in singly charged atomic form; (iii) $\zeta$, the cosmic-ray ionization rate; (iv) $n\left(\mathrm{H}_{2}\right)$. Note that $f_{\mathrm{D}}$ and $n\left(\mathrm{H}_{2}\right)$ can be estimated from observations (see Section 4), whereas the other two parameters have to be determined through the comparison between observed quantities and model results, in the same manner as $x(e)$. Variations in the above parameters allow us to cover the range of observed $\left[\mathrm{DCO}^{+}\right] /\left[\mathrm{HCO}^{+}\right]$and $\left[\mathrm{HCO}^{+}\right] /[\mathrm{CO}]$ abundance ratios in dense cores. Figure 2 shows these observed values in a sample of low mass cores (filled circles; from Butner et al. 1995), together with $x(e)$ and $\zeta$ contour level diagrams derived from model predictions for $n\left(\mathrm{H}_{2}\right)=10^{4} \mathrm{~cm}^{-3}, f_{\mathrm{D}}=2, T=10 \mathrm{~K}$, and at steady state (i.e. only model results at late times, $t>10^{6} \mathrm{yr}$, when steady state is reached, have been used). Note that the relatively low $\left[\mathrm{DCO}^{+}\right] /\left[\mathrm{HCO}^{+}\right]$ratio $(\sim 0.02)$ observed towards the cyanopolyyne peak of TMC-1 (open square, 


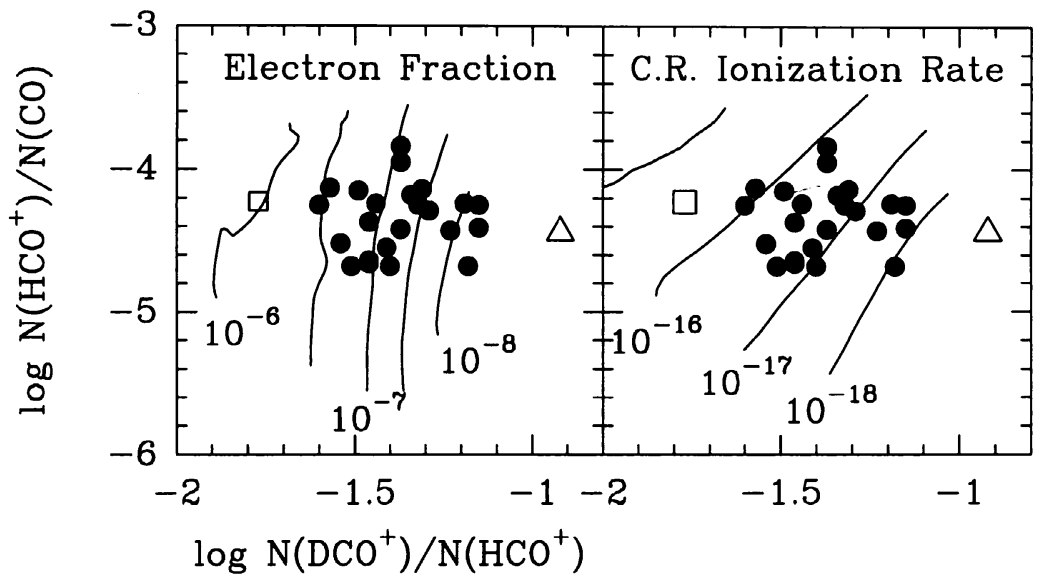

Figure 2. Observed $\left[\mathrm{HCO}^{+}\right] /[\mathrm{CO}]$ abundance ratios as a function of $\left[\mathrm{DCO}^{+}\right] /\left[\mathrm{HCO}^{+}\right]$in low mass cores. The curves represent contour level diagrams of (left) electron fraction and (right) cosmic ray ionization rate derived from detailed chemical models (Caselli et al. 1998).

Pratap et al. 1997) implies extremely high values of $x(e)$ and $\zeta$, although steady state models are probably not valid for this particular dense core (CWTH). The object with the highest $\left[\mathrm{DCO}^{+}\right] /\left[\mathrm{HCO}^{+}\right]$ratio is $\mathrm{L} 1544$ (open triangle), where $\left[\mathrm{DCO}^{+}\right] /\left[\mathrm{HCO}^{+}\right] \sim 0.12$ has recently been measured (Caselli et al. 1999). Judging from Fig. 2, L1544 seems to require a very low $x(e)$ and $\zeta$. However, the values of $f_{\mathrm{D}}$ and $n\left(\mathrm{H}_{2}\right)$ measured in this core are significantly higher than those assumed in the models used for Fig. 2.

A general approach to estimating the electron fraction, cosmic ray ionization rate, and metal abundances in low mass cores has been discussed by CWTH. One requires measurements of the $\left[\mathrm{DCO}^{+}\right] /\left[\mathrm{HCO}^{+}\right]$and $\left[\mathrm{HCO}^{+}\right] /[\mathrm{CO}]$ abundance ratios, the amount of depletion, and the volume density. A rough estimate of $f_{\mathrm{D}}$ can be obtained by using the $\left[\mathrm{HC}_{3} \mathrm{~N}\right] /[\mathrm{CO}]$ abundance ratio, which is strongly dependent on $f_{\mathrm{D}}$ and relatively independent of the cosmic ray ionization rate and refractory metal abundance (Ruffle et al. 1997; CWTH). Another approach is needed for massive cores and active star forming sites where the kinetic temperature may exceed $10 \mathrm{~K}$. Anderson et al. (1999) discuss this problem in detail and determine the degree of ionization across the $\mathrm{R}$ Coronae Australis core -where temperature gradients are measured through $\mathrm{CH}_{3} \mathrm{CCH}$ observations- using a simple chemical model where reactions with atomic deuterium are included.

To summarize, typical parameters found in low mass cores (excluding TMC1 and L1544, which will be separately treated in Section 4) are:

$$
\begin{array}{rll}
10^{-8} & \leq x(e) \leq & 10^{-6} \\
10^{-18} & \leq \zeta\left(\mathrm{s}^{-1}\right) \leq & 10^{-16} \\
3 \times 10^{-5} & \leq x(\mathrm{CO}) \leq & 7.5 \times 10^{-5}
\end{array}
$$




$$
1.2 \times 10^{-10} \leq \sum_{i} x\left(\mathrm{M}_{\mathrm{i}}^{+}\right) \leq 1.2 \times 10^{-7}
$$

The $x(\mathrm{CO})$ abundance range corresponds to values of $f_{\mathrm{D}}$ between 2 and 5 . The metal abundance $\sum_{i} x\left(\mathrm{M}_{\mathrm{i}}^{+}\right)$is a poorly constrained parameter, but the values obtained here corroborate the view of Graedel et al. (1982) that metals are highly depleted in dense cloud cores.

The new values of $x(e)$ can be used to investigate the magnetic stability of low mass cores against gravitational collapse. The timescale for ambipolar diffusion, whereby the neutral particles contract relative to the magnetic field and the ionized component, is $\sim 2.5 \times 10^{13} x(e)$ yr (e.g. Shu et al. 1987). In the sample considered by CWTH, the median core has a ratio between the ambipolar diffusion and free fall time scales equal to 50 , and hence, if their magnetic flux is indeed sufficient to prevent collapse, they will be much longer lived than suggested by their dynamical timescales. Similar conclusions have been reached using an independent data set by Williams et al. (1998).

In Orion massive cores, Bergin et al. (1999) found values of $x(e)$ which overlap with the low mass sources $\left(5.0 \times 10^{-8} \leq x(e) \leq 1.3 \times 10^{-7}\right)$ but have a lower median electron abundance of $6 \times 10^{-8}$. In still more massive cores associated with $\mathrm{O}$ and $\mathrm{B}$ clusters, typical electron fractions are $\sim 10^{-8}$ (de Boisanger et al. 1996). The majority of massive cores have $\left[\mathrm{DCO}^{+}\right] /\left[\mathrm{HCO}^{+}\right]<0.05$, indicative of gas temperatures $\sim 20 \mathrm{~K}$, which allow the reaction $\mathrm{H}_{2} \mathrm{D}^{+}+\mathrm{H}_{2} \rightarrow \mathrm{H}_{3}^{+}+\mathrm{HD}$ to proceed and lower the $\left[\mathrm{DCO}^{+}\right] /\left[\mathrm{HCO}^{+}\right]$ratio (see Sect. 2).

\section{The Starless Core L1544}

L1544 is a well known starless cloud core in Taurus. It has been intensely studied because observed molecular lines show asymmetric profiles indicative of extended inward motions (Tafalla et al. 1998; Williams et al. 1999; Ohashi et al. 1999). Thus, derived properties of this core may give us insight into the initial conditions which precede the collapse to form a star.

We have recently mapped L1544 in several molecular ions and CO isotopomers with the 30-m IRAM antenna, in order to study the electron fraction and its variation across the core. As noted in the previous section, an accurate estimate of $x(e)$ requires a measure of gas phase depletion $\left(f_{\mathrm{D}}\right)$. To determine $f_{\mathrm{D}}$, Caselli et al. (1999) compared $\mathrm{C}^{17} \mathrm{O}$ integrated intensity maps with $1.3 \mathrm{~mm}$ continuum dust emission from Ward-Thompson et al. (1999). They found that $f_{\mathrm{D}} \sim 10$ at the dust peak, and that observations are consistent with a model where $\mathrm{CO}$ is condensed out onto dust grains at densities above $\sim 10^{5} \mathrm{~cm}^{-3}$. The corresponding radius of the region where $\mathrm{CO}$ is severely depleted is $\sim 6500$ $\mathrm{AU}$ and the depletion causes $2.3 \mathrm{M}_{\odot}$ of gas to be lost to view in molecular line emission.

Preliminary results of our study on the electron fraction and the chemical structure of L1544 are reported in Figure 3 (left; Caselli et al. 2000), where fractional abundances of $\mathrm{DCO}^{+}, \mathrm{HCO}^{+}$, and electrons, together with the depletion factor $f_{\mathrm{D}}$, are displayed as a function of cloud radius. These results have been derived using a simple chemical scheme where the depletion of $\mathrm{CO}$ is followed as a function of time using a model for the source based upon the mm dust emission observations of Ward-Thompson et al. (1999). Ions such as 
$\mathrm{HCO}^{+}$are assumed to adapt instantaneously to the momentary $\mathrm{CO}$ abundance (see Caselli et al. 1999). The model is allowed to evolve until the $\mathrm{C}^{17} \mathrm{O}$ column density at the dust emission peak is consistent with that observed. Using CWTH models applied to observed abundance ratios, we derive a cosmic ray ionization rate of $\sim 3 \times 10^{-18} \mathrm{~s}^{-1}$. Introducing this value of $\zeta$ in the model, we find that $x(e)$ is a few $\times 10^{-9}$ at the dust peak, and is proportional to $n\left(\mathrm{H}_{2}\right)^{-0.5}$ over the density range $3 \times 10^{4}-10^{6} \mathrm{~cm}^{-3}$ in spite of the depletion at high density. The $\left[\mathrm{DCO}^{+}\right] /\left[\mathrm{HCO}^{+}\right]$abundance ratio increases inward with increasing depletion, and high density tracers peak in abundance in regions where $\mathrm{CO}$ is already considerably depleted, in agreement with observations.

High density tracers such as $\mathrm{HCO}^{+}$and $\mathrm{DCO}^{+}$are expected to be highly depleted at radii of less than $\sim 2800 \mathrm{AU}\left(\sim 20^{\prime \prime}\right.$ at the distance of L1544). Interestingly, Ward-Thompson et al. (1999) found that at $r<r_{\mathrm{f}}=2500 \mathrm{AU}$, the density distribution becomes flatter than a singular isothermal sphere (see also André et al. 1996), and the average volume density inside $r_{\mathrm{f}}$ is $n\left(\mathrm{H}_{2}\right)=1.5 \times 10^{6}$ $\mathrm{cm}^{-3}$. At these densities, a rough estimate of the "freeze-out" time scale for $\mathrm{CO}$ is $\sim 800 /\left(n_{6} S\right)$ years (!), with $n_{6}$ equal to the $\mathrm{H}_{2}$ number density in units of $10^{6} \mathrm{~cm}^{-3}$ and $S$ the sticking probability (thought to be of order unity, Jones \& Williams 1985; Tielens \& Allamandola 1987).

The presence of a "dense-depleted" region may be "visible" through high spectral resolution observations of optically thin high density tracers, which are likely to probe material at different velocities in front and to the rear of the depleted high density core, and thus to be asymmetric or "double" peaked (without however any preference for stronger blue or red peaks). All of this seems in general consistent with our observational results as shown in Figure 3 (right) which reports the first high sensitivity spectrum of $\mathrm{D}^{13} \mathrm{CO}^{+}$ever observed.

\section{Main Sources of Uncertainty}

An accurate estimate of the electron fraction implies measurements of $(\mathrm{i})\left[\mathrm{DCO}^{+}\right]$ $/\left[\mathrm{HCO}^{+}\right]$and $\left[\mathrm{HCO}^{+}\right] /[\mathrm{CO}]$ abundance ratios, (ii) $\mathrm{CO}$ depletion, (iii) density and temperature structure of the core. This implies accounting for the optical depth of observed lines and self-absorption effects in order to determine $x(e)$. One approach may be to observe rare isotopomers (e.g. $\mathrm{C}^{17} \mathrm{O}, \mathrm{HC}^{18} \mathrm{O}^{+}$, $\left.\mathrm{D}^{13} \mathrm{CO}^{+}\right)$in selected positions and use radiative transfer models to deduce physical parameters in the rest of the mapped core. As discussed in Sect. 4, the amount of gas phase $\mathrm{CO}$ depletion $\left(f_{\mathrm{D}}\right)$ can be estimated by comparing maps of optically thin $\mathrm{CO}$ isotopomers with millimeter continuum dust emission maps (Kramer et al. 1999; Caselli et al. 1999). Observations of the $\mathrm{HC}_{3} \mathrm{~N}$ molecule can also furnish rough estimates of $f_{\mathrm{D}}(\mathrm{CWTH})$.

Together with the above quantities, which can be deduced from observations, other parameters need to be considered when estimating the electron fraction in molecular clouds:

Metal depletion. - Species with low ionization potential such as metals, if not severely depleted, may significantly contribute to the degree of ionization in molecular clouds. However, atomic species such as $\mathrm{Fe}, \mathrm{Mg}, \mathrm{Na}$ cannot be directly detected in regions of high visual extinction. Thus, the gas phase abundance of metals and metal depletion has to be derived from chemical models 

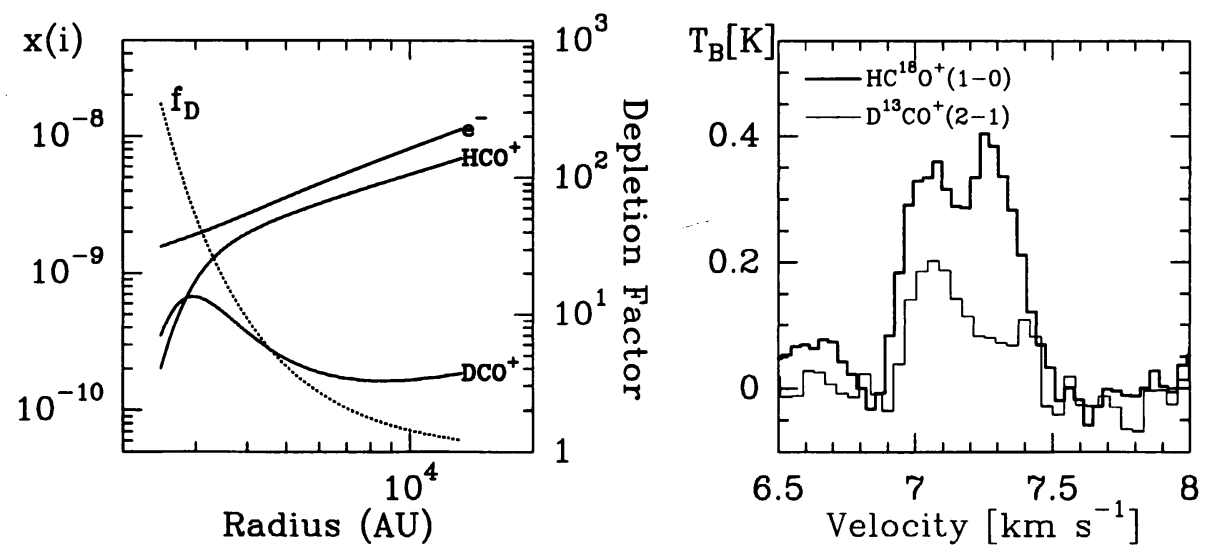

Figure 3. (Left) Radial distribution of $\mathrm{DCO}^{+}, \mathrm{HCO}^{+}$, and electron fractional abundances, and the depletion factor $f_{\mathrm{D}}$, in L1544 as predicted by a simple chemical model where the freeze-out of gaseous species onto dust grains is taken into account. (Right) Spectra of $\mathrm{D}^{13} \mathrm{CO}^{+}(2-1)$ and $\mathrm{HC}^{18} \mathrm{O}^{+}(1-0)$ towards the dust peak in $\mathrm{L} 1544$, observed at the 30-m IRAM antenna. The presence of two components in optically thin high density tracers is an indication that these species are depleted out in the densest part of the core (see text).

and observations of high density tracers (e.g. CWTH). Note that CWTH found the $[\mathrm{CCS}] /[\mathrm{CO}]$ ratio to be sensitive to the abundance of refractory metals (see their Fig. 6).

Cosmic ray ionization rate. - Substantial variations in the cosmic-ray induced ionization rate $(\zeta)$ were found when early models of the diffuse molecular clouds were developed (Hartquist et al. 1978). Moreover, both theoretical (e.g. Hartquist \& Morfill 1983,1994) and experimental (Fukui \& Hayakawa 1981) work has shown that the bulk of the ionizing cosmic rays have energies in a range (1 to $16 \mathrm{MeV}$ ) that could cause ionization losses to be significant through modest column densities of material. Therefore, the ionization rate at any particular point may depend sensitively on the distribution of surrounding gas. It also seems likely that the presence of a nearby supernova remnant or possibly a wind-blown bubble will significantly enhance the ionization rate in many cases. Alternatively, modulation of the cosmic rays by a high velocity flow, as occurs in the Solar System (e.g. Fisk \& Wenzel 1998), may lead to decreases in the ionization rate in some places. As in the case of metal abundances, comparisons between detailed chemical models and molecular line observations are needed to estimate $\zeta$. The $\left[\mathrm{C}_{3} \mathrm{H}_{2}\right] /[\mathrm{CO}]$ abundance ratio seems to be especially sensitive to the cosmic ray flux (CWTH).

Grain/PAH recombination coefficient - Lepp \& Dalgarno (1988) found that for abundances of Polycyclic Aromatic Hydrocarbons (PAHs) relative to hydrogen greater than $10^{-8}$, the free electrons attach to PAH molecules to form $\mathrm{PAH}^{-}$negative ions, although the effective sticking depends on electron affini- 
ties as well as the fraction of $\mathrm{H}$ atoms in PAH's (e.g. Allamandola et al. 1989). In this case, the $\mathrm{PAH}^{-}$will carry most of the electric charge, and interstellar chemistry is changed qualitatively as atomic and molecular ions undergo nondestructive mutual neutralization reactions with $\mathrm{PAH}^{-}$. However, in dense clouds the PAH molecules will probably collide to form larger molecular systems, and they will also condense onto grains. Chemical models will have to include PAHs and single out observable species which are most sensitive to PAH abundance.

$X$-Rays - Einstein Observatory, ROSAT, and ASCA observations of star forming regions have shown that young stellar objects are strong emitters at $\mathrm{X}$-ray wavelengths. Most classical and weak lined T Tauri stars in several star forming regions have been detected as X-ray sources (e.g. Feigelson \& Montmerle 1999). X-ray emission has recently been detected also from Class I protostars (Grosso et al. 1997; Ozawa et al. 1999). Lepp \& Dalgarno (1996) discuss X-rayinduced chemistry of interstellar clouds and predict the $[\mathrm{HCN}] /[\mathrm{CO}]$ ratio, $\mathrm{CN}$ and NO abundances to increase with increasing ionization rate. In star-forming regions, detailed observational studies of appropriate species are required in order to estimate the contribution of $\mathrm{X}$-rays to the ionization rate.

UV photons. - UV photons are particularly important for the charge balance of (i) cloud envelopes, where the visual extinction $A_{\mathrm{V}}$ may drop to values less than 3 mag (McKee 1989; Hartquist et al. 1998), and (ii) cores with clumpy structure, where UV can penetrate into the innermost and densest regions (e.g. Myers \& Khersonsky 1989). The $\left[\mathrm{HCO}^{+}\right] /[\mathrm{CO}]$ abundance ratio increases with decreasing $A_{\mathrm{V}}$ (Turner 1995; Lucas \& Liszt 1996). Observations of weak emission from $\mathrm{HCO}^{+}$and $\mathrm{CO}$ outside cores, in gas with density $\sim 10^{3} \mathrm{~cm}^{-3}$, in addition to stronger emission in the denser gas will furnish information on the ionization structure both in and around cores.

In summary, comprehensive chemical models, including time dependent gas phase depletion, need to be applied to detailed molecular line observations in order to infer the degree of ionization in molecular clouds. In the future, we plan to include gas-grain interactions in our models, and extend the work undertaken for L1544 in other objects, to build detailed $n\left(\mathrm{H}_{2}\right)$ and $x(e)$ maps in a significant sample of cloud cores. This is fundamental for a better understanding of core properties and the whole process of star formation.

Acknowledgments. I am grateful to all my collaborators, and to Tom Hartquist, Eric Herbst and Malcolm Walmsley for carefully reading the manuscript.

\section{References}

Allamandola, L.J., Tielens, A.G.G.M., \& Barker J.R. 1989, ApJS, 71, 733 Anderson, I.M., Caselli, P., Haikala, L.K., \& Harju J. 1999, A\&A, 347, 983

André, P., Ward-Thompson, D., \& Motte, F. 1996, A\&A, 314, 625

Bergin, E.A., Plume, R., Williams, J.P., \& Myers P.C. 1999, ApJ, 512, 724

Butner, H.M., Lada, E.A., \& Loren, R.B. 1995, ApJ, 448, 207

Caselli, P., Walmsley, C.M., Tafalla, M., Dore, L., \& Myers, P.C. 1999, ApJ, $523, \mathrm{~L} 165$

Caselli, P., Walmsley, C.M., Terzieva, R., \& Herbst, E. 1998, ApJ, 499, 234 $(\mathrm{CWTH})$ 
Caselli, P., Walmsley, M., Galli, D., Tafalla, M., \& Myers, P.C. 2000, in preparation

Dalgarno, A. \& Lepp S. 1984, ApJ, 287, L47

de Boisanger, C., Helmich, F.P., \& van Dishoeck, E.F. 1996, A\&A, 310, 315

Feigelson, E.D. \& Montmerle, T. 1999, ARA\&A, 37, 363

Fisk, L.A. \& Wenzel, K.-P. 1998, Space Science Reviews, 83, 179

Fukui, Y. \& Hayakawa, S. 1981, in 17th International Cosmic Ray Conference, 2,226

Graedel, T.E., Langer, W.D., \& Frerking M.A. 1982, ApJS, 48, 321

Grosso, N., Montmerle, T., Feigelson, E.D., André, P., Casanova, S., \& GregorioHetem, J. 1997, Nature, 387, 56

Guélin, M., Langer, W.D., \& Wilson, R.W. 1982, A\&A, 107, 107

Hartquist, T.W., Caselli, P., Rawlings, J.M.C., Ruffle, D.P., \& Williams, D.A. 1998, in The Molecular Astrophysics of Stars and Galaxies, eds. T.W.

Hartquist \& D.A. Williams, Clarendon Press, Oxford, 101

Hartquist, T.W., Doyle, H.T., \& Dalgarno, A. 1978, A\&A, 68, 65

Hartquist, T.W. \& Morfill, G.E. 1983, ApJ, 266, 271

- 1994, Ap\&SS, 216, 223

Herbst, E. \& Leung, C.M. 1989, ApJS, 69, 271

Jones, A.P. \& Williams, D.A. 1985, MNRAS, 217, 413

Kramer, C., Alves, J., Lada, C.J., Lada, E.A., Sievers, A., Ungerecths, H., \& Walmsley, C.M. 1999, A\&A, 342, 257

Larsson, M., et al. 1996, A\&A, 309, L1

Lee, H.-H., Bettens, R.P.A., \& Herbst, E. 1996, A\&AS, 119, 111

Lepp, S. \& Dalgarno, A. 1988, ApJ, 324, 553 1996, A\&A, 306, L21

Linsky, J.L., Diplas, A., Wood, B.E., Brown, A., Ayres, T.R., \& Savage, B.D. 1995, ApJ, 451, 335

Lucas, R. \& Liszt, H. 1996, A\&A, 307, 237

McCall, B.J., Geballe, T.R., Hinkle, K.H., \& Oka, T. 1999, ApJ, 522, 338

McKee, C.F. 1989, ApJ, 345, 782

Millar, T.J., Bennet, A., \& Herbst, E. 1989, ApJ, 340, 906

Myers, P.C. \& Khersonsky, V.K. 1995, ApJ, 442, 186

Ohashi, N., Lee, S.W., Wilner, D.J., \& Hayashi, M. 1999, ApJ, 518, L41

Ozawa, H., Nagase, F., Ueda, Y., Dotani, T., \& Ishida, M. 1999, ApJ, 523, L81

Pratap, P., Dickens, J.E., Snell, R.L., Miralles, M.P., Bergin, E.A., Irvine, W.M., \& Schloerb, F.P. 1997, ApJ, 486, 862

Ruffle, D.P., Hartquist, T.W., Taylor, S.D., \& Williams, D.A. 1997, MNRAS, 291,235

Shu, F.H., Adams, F.C., \& Lizano, S. 1987, ARA\&A, 25, 23

Tafalla, M., Mardones, D., Myers, P.C., Caselli, P., Bachiller, R., \& Benson, P.J. 1998, ApJ, 504, 900

Tielens, A.G.G.M. \& Allamandola, L.J. 1987, in Interstellar Processes, eds. D.J. Hollenbach \& H.A. Thronson, Jr. (Dordrecht: Kluwer), 397

Turner, B.E. 1995, ApJ, 449, 653

Watson, W.D. 1977, CNO Isotopes in Astrophysics, ed. J. Audouze, Reidel Publ. (Dordrecht)

Ward-Thompson, D., Motte, F., \& André, P. 1999, MNRAS, 305, 143

Williams, J.P., Bergin, E.A., Caselli, P., Myers, P.C., \& Plume, R. 1998, ApJ, 503,689 
Williams, J.P., Myers, P.C., Wilner D.J., \& Di Francesco, J. 1999, ApJ, 513, L61

Wootten, A., Loren, R.B., \& Snell, R.L. 1982, ApJ, 255, 160

Wootten, A., Snell, R.L., \& Glassgold, A.E. 1979, ApJ, 234, 876

\section{Discussion}

T. Geballe: Values for $\zeta$ in the literature range from $\sim 1 \times 10^{-17}$ to $5 \times 10^{-17}$. Your models give a much wider range $\left(10^{-16}-10^{-18}\right)$. How realistic are such large and small values?

P. Caselli: Significant variations in the cosmic may induced ionization scale (between $10^{-18}$ and $10^{-16}$ ) were already required in early models of the diffuse molecular clouds (e.g. Hartquist, Doyte, \& Dalgarno 1978). Moreover, both theoretical (e.g. Hartquist \& Morfill 1983) and experimental work (e.g. Fukui \& Hayakawa 1981) have shown that the bulk of the ionizing cosmic rays have energies in a range ( 1 to $16 \mathrm{MeV}$ ) that could cause ionization losses to be significant through modest column densities of material. Magnetic field geometry may play an important role. It also seems likely that the presence of a nearby supernova remnant or a wind-blown bubble will significantly enhance the ionization rate in many cases.

J. Rawlings: The dynamics of L1544 are very complex. In Tafalla et al. (1998) it was pointed out that since the spatial extent of the region generating spectral asymmetry is large, yet the core is young, the Shu (1977) model is inappropriate. Similarly, the lack of ion-neutral drift together with the relatively large line widths suggests that ambipolar diffusion models are not applicable. Have there been any developments in our understanding?

P. Caselli: There have been no developments in understanding the dynamics in L1544. Tafalla et al. (1998) pointed out that in L1544 there is a global pattern of inward motions (background and foreground layers approaching each other). They deduce that while the foreground gas has a constant velocity, the background material presents systematic velocity changes interpreted as arising from two velocity components. After the new obseravations carried out at the $30 \mathrm{~m}$ IRAM antenna, we think that the two velocity components observed in optically thin lines (e.g. $\left.\mathrm{D}^{13} \mathrm{CO}^{+}(2-1)\right)$ towards the dust peak are due to the existence of a depleted high density core so that only material at different velocities in front of and to the rear of the depleted core is detected.

O.M. Shalabiea: Do not we also need to consider the depletion during the evolutionary time of chemical models?

P. Caselli: Yes, we should include time-dependent depletion in our models to more realistically determine the electron fraction in dense cloud cores. The main problem is that there are still many uncertainties regarding desorption mechanisms and sticking probabilities which may only add other free parameters to the model. However, the next step will be to use "modified" gas-grain chemical models with deuterium chemistry to reproduce the observed $\left[\mathrm{HCO}^{+}\right] /[\mathrm{CO}]$ and $\left[\mathrm{DCO}^{+}\right] /\left[\mathrm{HCO}^{+}\right]$abundance ratios and derive the electron fraction, cosmic ray ionization rate, and metal abundances. 